ReVista DE Filosofía

Volumen 71 (2015) 9-21

\title{
EL PROBLEMA DE LA DETERMINACIÓN DEL "SABER" EN DIÁLOGOS TEMPRANOS DE PLATÓN
}

\author{
Francisco Abalo $C$. \\ Universidad de Chile \\ fjabalocea@gmail.com
}

\begin{abstract}
Resumen / Abstract
El siguiente trabajo se enmarca en una investigación acerca de las fuentes históricofilosóficas que dan cuenta de los fundamentos de la tendencia de la filosofía a interpretarse a sí misma como una ciencia (saber). Hemos tomado algunos de los diálogos socráticos de Platón para pesquisar cómo opera esto ahí, cuál es su motivación última y cuál su sentido. El supuesto básico es que cabe, en algún sentido, pensar la actividad filosófica como un "saber del saber". El hilo conductor lo constituye el examen de ciertos pasajes ejemplares en donde aparece la llamada "analogía del arte", a través de la cual se ponen al descubierto ciertos caracteres esenciales del saber. Finalmente se concluye que la motivación de un "saber referido al saber" no puede ser "epistemológica" sino fundada en la preocupación por alcanzar una vida de excelencia.
\end{abstract}

Palabras ClaVe: Platón, Sócrates, diálogos socráticos, saber, la analogía del arte.

THE PROBLEM OF THE DETERMINING THE “WISDOM” IN PLATO'S EARLY DIALOGUES

This article is part of an investigation into the historical and philosophical sources that explain the tendency of the philosophy to interpret itself as a science (wisdom). We have taken some of the Plato's early dialogues to explore how this tendency works there, what its ultimate motivation and what its meaning. The basic assumption is that the philosophy, in some sense, is "knowledge of the knowledge". The way of the inquiry is the examination of remarkable passages where the "analogy with the art" appears. This analogy exposes certain essential characteristics of wisdom. Finally we conclude that the motivation of the "knowledge of the knowledge"cannot be "epistemological" but based on the concern to achieve a life of excellence.

KEY WORDS: Plato, Socrates, Socratic dialogues, wisdom, the analogy of art.

Consideración general

Da presente exposición está motivada en líneas muy generales por una de esas preocupaciones centrales para la así llamada "fenomenología hermenéutica". En concreto, me refiero a ese afán que se hace tan nítido en el pensamiento del joven 
M. Heidegger ${ }^{1}$ y que consiste en poner en el centro de la búsqueda una determinación apropiada de la filosofía partiendo de las experiencias fundamentales que la mueven.

El segundo aspecto que inspira mi intento consiste en asumir esa clara conciencia sobre la relevancia filosófica de la "historia de la filosofía". La interpretación críticohermenéutica del legado de la tradición rebasa el afán meramente anticuarial y constituye una vía fértil para proyectar el ejercicio filosófico hacia adelante.

\section{Presentación del problema y articulación general de su desarrollo}

Partamos con la siguiente pregunta: ¿es casual en el contexto de la tarea aludida el nombre de Platón? Por lo pronto solo podemos dar un indicio que nos introduce en el tema. Éste concierne a lo problemático que resulta a la sazón el hecho de que la filosofía haya buscado una determinación de sí misma como "ciencia". Esto se ha vuelto, por lo menos hoy, nada de evidente. Cierto es que esta tendencia histórica de la filosofía no es unívoca, y esto porque lo que se entiende en cada caso por "ciencia" no es idéntico. No es lo mismo lo que Aristóteles nombra como "ciencia primera" que "LA ciencia" que mienta Hegel. Es razonable pensar, no obstante, que hay ciertas conexiones internas y que, por otra parte, la filosofía justamente ha sido en este afán uno de los agentes principales en la mutación del concepto de "ciencia".

¿Es Platón un hito significativo o incluso inicial en esta tendencia? Si es así, ¿se han perpetuado las direcciones originales de esta orientación? ¿Ha sido superado en sus direcciones básicas? Estas preguntas, de ser hoy atingentes y útiles, suponen por lo menos hacerse cargo de eso que podemos constatar en algunos diálogos platónicos como direcciones dominantes con respecto a esta tendencia de la filosofía. En otras palabras, la pregunta inicial es: de qué manera y en qué sentido esta tendencia de la filosofía a autointerpretarse como "ciencia" tiene lugar en Platón. Pero a la vez esta pregunta va amarrada a esta otra: ¿en qué medida y cómo en este mismo acto se ponen de realce rasgos decisivos que caracterizan por primera vez de forma expresa eso que llamamos "ciencia"?

Sin embargo, con respecto a estas preguntas, las siguientes consideraciones solo pueden ser parciales; principalmente porque tomaremos como hilo conductor ese recurso explicativo en Platón que los estudiosos han nombrado "la analogía con el arte"2. Esto restringe el intento a esos diálogos del período temprano de Platón, o los llamados

1 Me refiero sobre todo a la lección titulada Phänomenologische Interpretation zu Aristoteles (Heidegger 1985). Tal vez no esté demás advertir que la mención de Heidegger en este contexto opera en relación con direcciones más bien generales del intento y no inciden, directamente al menos, en las consideraciones concretas de este trabajo.

2 Al menos es nombrada así ("analogy with the arts and science") por CH. Kahn (Kahn 1996, p. 129). Si bien esta analogía no se identifica exactamente con el método inductivo 
también "socráticos", en donde la "analogía" tiene una presencia más evidente. De esta forma intentaremos apuntar a lo anterior mediante tres preguntas concretas: 1) ¿Cuál es el sentido general y las principales funciones de dicha "analogía”? 2) ¿Cuáles son los rasgos esenciales de la "ciencia" que podemos descubrir mediante esta analogía? 3) ¿Cuáles son los problemas y las posibilidades en relación con la autodeterminación de la filosofía que se siguen de aquí?

Sentido y funciones de la analogía del arte en los diálogos tempranos de Platón

Nuestra palabra "ciencia" traduce normalmente el vocablo griego epistemê. Y si bien esta traducción se ajusta en general, en el caso de Platón resulta restringida ${ }^{3}$. El punto principal reside en que, para Platón, epistemê es término perfectamente intercambiable con sophia (sabiduría) o incluso, lo que es verdaderamente significativo, con technê (arte, técnica). En consecuencia, resulta al menos sesgado traducir en todo contexto epistemê por "ciencia", si con ello pensamos predominantemente en un comportamiento de tipo teórico, como en la biología o la física actuales, a diferencia de un saber "meramente técnico". En cambio, para Platón un "arte" como la zapatería o la construcción de casas son "ciencia", así como la aritmética o la astronomía son techne 4 . Por eso, a fin de evitar malentendidos, en adelante hablaremos más bien de "saber", por ser más amplio e indeterminado, y de esta forma recoger el parentesco semántico desde el que Platón habla indistintamente de epistemê, technê o sofia ${ }^{5}$.

¿En qué consiste, por tanto, la “analogía con el arte”? Ya con esto nos enfrentamos a un problema que dificulta una respuesta inmediata. Pues si quisiéramos decir, siguiendo la apariencia general, que consiste en un recuento bastante flexible de una serie de "saberes" (sobre todo del tipo técnico-productivo, aunque no solo ${ }^{6}$ ) que nos permite inferir "algo más general"; esta caracterización resulta lo suficientemente vaga como para dejar indeterminado "eso" para lo que se introduce este recurso socrático-platónico.

(epagogê) que se le atribuye a Sócrates desde antiguo (Euclídes), si se puede conceder que la analogía del arte es al menos una forma específica de éste.

$3 \quad$ Así por ejemplo en Kahn 1996, p. 126.

4 Hago notar solo al paso que para nosotros (los de habla castellana en Chile) no siempre "ciencia" quiere decir algo como lo descrito. Así, se conserva un sentido más "pragmático" de ciencia en giros como "algo tiene su ciencia", es decir, tiene su forma apropiada de trato.

5 Tal vez si se quisiera ser más preciso cabría nombrarlo, siguiendo cierto uso en Platón (Ión 532 c5), comportamiento "técnico-espitémico".

6 Es notable que en muchas de las listas de "saberes" que expone Sócrates destaque la aritmética; así por ejemplo en Ión (531 e5) o en un notable pasaje del Gorgias (450 d4 ss.) en el que la aritmética es tomada como modelo de "arte" (techne) cuya obra se lleva a cabo enteramente a través de discursos (razonamientos; logoi) a diferencia de las que manufacturan algo. 
Incluso dejando de lado el problema del "inductivismo" en la analogía del arte , la cuestión es a mi entender que esta "analogía" depende en su sentido del contexto en que es introducida en los diversos pasajes y que, por tanto, su propósito y función no es unívoco. De esta manera, es necesario distinguir por lo menos en términos gruesos estas funciones específicas. Sin embargo, tal distinción parte por comprender los supuestos básicos que animan su aplicación, es decir, el sentido general que la posibilita.

Tomemos primero lo de su sentido general. La base de la aplicabilidad la podemos encontrar ya en un célebre pasaje (20c3-23c) de la Apología de Sócrates. Me explico: la introducción en diversos contextos dialógicos de dicha analogía se entiende, en parte, por el alto valor que se le concede ahi a ese comportamiento especifico que podemos llamar "saber", y que en definitiva no es sino el reflejo de la eminencia que tiene esta forma de comportamiento entre los griegos en general. "Tener un saber", "ser sabio" no es para un griego cualquier cosa. Solo sobre la base de esta valoración del saber se puede entender la total extrañeza y la consecuente insistencia de Sócrates que llega a conformar su propio destino vital al intentar comprender ese oráculo délfico que lo declara "sabio en sumo grado" (21b5).

Ahora bien, este mismo pasaje aporta otros aspectos más directamente conectados con la "analogía".

En primer lugar, resulta patente en este breve relato biográfico que la "investigación" socrática del sentido del oráculo apunta al descubrimiento de que la certeza de no creer saber lo que no sabe es precisamente saber en grado sumo y que, por consecuencia, es posible la descalificación de otras actividades que solo pretenden ser un saber (21d). De esta forma, lo que ya este pasaje destaca es que tanto el "saber" como su opuesto, la "ignorancia", no solo se juegan en una relación directa a un objeto de conocimiento, es decir, en donde saber es saber directamente "algo" (un objeto) e ignorarlo es directamente no saberlo. Más bien cabe hablar de un "saber" que es consciente de que ignora algo (la "sabiduría" socrática); pero a su vez encontramos una segunda forma de ignorancia, más pertinaz que la anterior, en la que solo se cree saber, pues lo que se ignora es precisamente el hecho de ignorar algo. Esto último por lo menos se cumple en sentido absoluto en los dos primeros arquetipos (políticos y poetas). En el último caso, los "artesanos", es decir, los que efectivamente son entendidos en un campo objetual

7 Acerca del problema del inductivismo presente en la analogía con el arte se puede consultar Kahn 1996, pp. 191-194. Lo interesante del análisis es la detección de los límites de este método en el contexto del examen de la posibilidad de un "saber del saber", tal como se da esta aporía en el Cármides. Los descubrimientos expuestos ahí han sido especialmente fructíferos para el desarrollo de este trabajo. Me parece, en todo caso, que en el método inductivo persiste el problema de que si el recuento de casos puede hacerse sin tener a la vista lo general, o si al revés solo sobre la base de algo general puede hacerse este recuento (¿de qué forma está esto "general" desde el principio?). Ahora bien, cabe pensar que el orden "pedagógico" en que al interior de los diálogos se "infieren inductivamente" los aspectos generales no es necesariamente el orden de primacía epistemológica y ontológica de lo general con respecto a los casos. 
concreto, Sócrates les concede el estatuto de "sabios", aun cuando sea precisamente su saber lo que los hace también ignorantes en el segundo sentido, pues su ignorancia no consiste sino en desconocer los límites de su saber y así estimarse desmesuradamente como "sabios" "también en las demás cosas, incluso en las más importantes..." (22 d6).

En segundo lugar, a pesar del marcado "escepticismo" que aquí exhibe Sócrates, es sostenible en principio que para detentar esta posición (eminentemente aporética) es necesario, por lo menos de forma implícita, disponer de algunos criterios mínimos para diferenciar en general lo que es saber de lo que no lo $e^{8}$. Y precisamente la analogía con el arte hace visibles estos caracteres del saber en general, los cuales justamente nos permiten discriminar qué puede calificar y qué no para ser considerado así. Dicho de modo tentativo: una posición como la socrática, es decir, la filosófica, pareciera suponer por lo pronto algo así como un "saber del saber y del no saber"en el respecto aludido. La formulación no es casual; la encontramos en el Cármides (1665) así tal cual y constituye un agudo problema como posibilidad de autointerpretación de la filosofía.

Ahora bien, ¿cuáles son las motivaciones concretas que obligan a Platón a hacer uso de esta "analogía"? Esto solo lo podemos comprender si echamos una rápida ojeada a ciertos diálogos y pasajes en los que aparece concretamente eso a lo que apunta en cada caso la analogía.

Uno de los primeros diálogos en que la analogía aparece de forma muy marcada es el Ión. En él, la conversación se desarrolla entre Sócrates y un rapsoda, y tiene por asunto principal discernir si lo que éste hace y, en definitiva, de lo que depende en última instancia este quehacer (la poesía) pueden ser considerados un "arte". La posición socrática es clara: ni el rapsoda ni el poeta dicen lo que dicen "por arte" (534 d ss.). Ahora bien, si descontamos la explicación positiva de Sócrates acerca del origen "divino" de la poesía ( 534 d-536 d3), casi toda la fuerza argumentativa se sustenta en la comparación de esta actividad con saberes como la adivinatoria, la aritmética, la medicina, la escultura, entre otros. ¿Cuál es aquí la función de la analogía? Resulta bien evidente: la actividad del rapsoda no puede calificar para ser considerada "saber". Primero, como el mismo Ión es testimonio vivo de esto (cfr. $532 \mathrm{c} 4$ ), no es propio de un entendido no ser capaz de comprender lo dicho por todos los poetas en cuanto todos refieren a las mismas cosas y, por tanto, solo quien como un entendido está referido directamente a esas "cosas" de las que hablan los poetas puede discernir quién de entre ellos habla "más bellamente" y quién no (531c-532c2). En segundo lugar, porque de entre las muchas cosas de las que habla, por ejemplo Homero, ninguna concierne directamente al rapsoda en cuanto tal, habiendo sí muchas de ellas en las que es solo un entendido específico el que será capaz de entender y discernir (537 d1-4). En definitiva, aquí la

8 Si bien es cierto que este reconocimiento expreso no se le puede atribuir sin más a Sócrates, que parece haber procedido de forma harto más espontánea, sí se lo puede encontrar en el desarrollo del Sócrates platónico. Aquí no pretendo ni rozar la tan famosa "cuestión socrática", de la cual hay ya abundante bibliografía. Para la introducción a este problema se puede consultar con provecho Gómes-Lobo 1998; también Guthrie 1998 y Vlastos 1991. 
función descalificadora de la analogía funciona sobre la base de un carácter concreto con el que debe cumplir todo "saber": todo "saber" es constitutivamente "específico", esto es, referido a un determinado campo de objetos.

Otro diálogo en el que la analogía funciona de una forma visible es en el Gorgias, fundamentalmente al menos en la parte en que tiene lugar la conversación de este orador con Sócrates. La función que cumple aquí la analogía es hasta cierto punto semejante al contexto anterior. Pues, la insistencia con la que Sócrates pregunta por el objeto específico de este pretendido saber y el fracaso reiterado del retórico por responder satisfactoriamente, deja entrever que al igual que, como antes la poesía, la retórica no califica tampoco por este lado con esta condición mínima. Sin embargo, el paralelismo llega hasta cierto punto, ya que en el fondo la retórica no solo pretende ser un cierto saber, sino que su supuesto poder consiste en "abarcar y tener bajo suyo las potencias de todos los saberes"( 456 b) en tanto su objeto propio es "el más grande y elevado de los asuntos humanos" (451 d6-7). De esta manera, sobre la base de una analogía más compleja que presenta a las claras la conexión entre "saber" y "bien" (464 b2-466 a4), lo que está en juego no es solo refutar la pretensión de que la retórica sea un "saber", sino la desmesurada pretensión de que sea el "bien supremo" en tanto se considera como el "saber" que rige por sobre el resto y con ello, los destinos de la polis.

El tercer contexto que he escogido difiere ostensiblemente de los dos anteriores. En efecto, encontramos un notable ejemplo de esta analogía en un pasaje del Laques (189 d4-190 b). Justamente en este pasaje, Sócrates propone un indagación "más fundamental" (189 e2) para reconocer quién de entre los que conversan califica para dar consejo acerca de cómo habrán de ser educados los jóvenes para llegar a ser "los mejores". Estas condiciones fundamentales son el índice de que estamos en presencia de un "entendido en el cuidado de las almas" (185 e-6). Aquí la comparación se establece, como en muchos otros lugares, en relación con la medicina9. Todas aquellas condiciones fundamentales que hacen de un médico un entendido en el cuerpo, es lo que se pide que cumpla ese experto en almas. Lo decisivo estriba, por tanto, en que aquí la base analógica no intenta deslegitimar un pretendido "saber", sino establecer las condiciones de uno al menos posible; a saber, de un posible saber que trata de eso que cualquiera estima "mucho más que el cuerpo", pues es justamente aquello "de lo que depende que todos tus asuntos se hagan bien o mal, volviéndote en eso tú mismo valioso o miserable", como dice Sócrates del "alma" en el Protágoras (313 a7-8).

9 Es notable en el contexto de estos diálogos socráticos la relevancia del arte médico. Si bien se ha sostenido, con razón, a mi juicio, que en Platón la matemática (aritmética y geometría) constituye un modelo epistémico, en estos diálogos tempranos se puede vislumbrar que en otro respecto es la medicina un modelo técnico-epistémico. No solo en este pasaje aludido del Laques, sino que, por ejemplo, en el Protágoras aparece el giro "médico del alma" (peri tên psuchên iatrikos; 313 e2) atribuido a alguien que es capaz de discernir de entre toda la "mercancía sofistica" qué es útil y qué nocivo para el alma. En la misma dirección se encuentra la analogía entre el arte médico y la justicia en el Gorgias (478 a-479 c5). 


\section{Algunos caracteres de la determinación platónica del saber}

Ahora quisiera avanzar hacia algunos de los caracteres esenciales del saber que se hacen visibles a través de estos tres contextos. Sin embargo, antes de adentrarnos en esto me permito una consideración general.

Se puede percibir con relativa nitidez, tanto en estos pasajes como en otros, que la motivación principal de Platón no está puesta primeramente en conformar una rudimentaria "epistemología". Si nos fijamos con atención lo que ahí está puesto en juego, indirectamente al menos, es precisamente un posible y legítimo saber acerca de aquellas disposiciones que hacen mejor al "alma"(las virtudes), una "epistemê sobre el bien y el mal", como la llama en el Cármides, o una verdadera "política", como en el Protágoras o en el Gorgias. En efecto, la conformación de un tal tipo de "ciencia" es un motivo central en el pensamiento platónico, cuya exposición más vasta y honda la encontramos por lo pronto en la Politeia, pero que incluso la podemos encontrar como motivación vital del propio Platón en algunas de sus aventuras biográficas. En este sentido es penetrante la observación de Kahn (1996, p. 104), según la cual "en orden a establecer la existencia de una technê moral, Platón tiene que aclarar primero la noción de technê"'. De este modo, es justamente la eminencia de este motivo lo que explica la pertinencia de las consideraciones "epistemológicas". Pero, se puede llevar la cosa más allá y afirmar que la dimensión "psicológica" e incluso la ontológica del pensamiento platónico tienen en esta preocupación básica su punto de arranque. En efecto, es una clave interpretativa muy fecunda considerar que precisamente el problema del estatuto ontológico de entidades tales como las "virtudes"es lo que lleva al pensamiento platónico a una dimensión metafísica de inmensos alcances y efectos para la posteridad.

Dicho esto, pasemos ahora a los caracteres.

El primer carácter parece obvio a primera vista, sin embargo es completamente decisivo para la determinación más plena. Pues parece una constatación evidente que todo saber refiera a un objeto, o mejor, a un campo de objetos bien determinado, y que, recíprocamente, dicho campo de objetos sea conocido solo por un saber determinado y no por otro. Pero si se mira con cuidado lo que Platón apunta con esto, esta "evidente constatación" es justamente la base desde la que la determinación se va problematizando. ¿Por qué? Porque (como Sócrates los destaca) ya el nombre de un saber se decide a partir de la determinación del campo de objetos al que se refiere. Ya aquí podemos encontrar al menos dos problemas, de los que creo Platón toma conciencia, a saber, 1) si esta "determinación" de un campo de objetos los reúne solo según su extensión o si un mismo campo objetual puede ser referido de forma "intencionalmente" 10 diversa; 2) se hace inevitable la pregunta por el origen y el modo de obtener esta "determinación".

10 Creo que es necesario señalar el uso metodológico que aquí hago de este par: "extensional”"intencional" (de intentio y no de intensio). No es coincidente con el uso lógico de extensiónintensión (comprensión/connotación). La oposición que aquí introduzco es más cercana, si 
En el Gorgias se desarrollan ciertas cuestiones contenidas en lo anterior. De esta forma, al inicio de la conversación con Polo se puede ver cómo en el fondo el campo objetual determinado no es referido solo y en primer lugar según su extensión, sino de forma intencionalmente diferente. Si esto es así, entonces esta perspectiva nos obliga a mirar y caracterizar este referencialidad del saber de forma más atenta. Pues justamente hay una serie de comportamientos orientados a ciertos objetos (cuerpo, alma) que se hacen pasar por saberes, y que incluso intentan suplantarlos, pero que no constituyen sino un conjunto de "destrezas" (empeiriai) cuyo fin es complacer y adular (462 b3-463 a4). A este conjunto pertenecen tanto la retórica como la sofística, en cuanto destrezas orientadas a la complacencia del alma, y análogamente la culinaria y la cosmética, en cuanto orientadas a la complacencia del cuerpo. Pero también al interior del conjunto de saberes cabe una distinción intencional, pues tanto gimnástica como medicina se orientan a un objeto común (la salud del cuerpo) en respectos distintos (por mantenerla o por producirla), así como análogamente política y justicia se orientan hacia un objeto común (el alma) en respectos diversos (por regular el comportamiento o corregirlo). Ahora bien, quedándonos solo en la diferencia intencional entre "destrezas adulatorias" y "saberes", aparecen nuevos caracteres de estos últimos. Por lo pronto, que es solo un saber lo que puede estar orientado hacia el "bien" de su objeto, es decir, es capaz de disponer ese objeto del mejor modo (beltistion) y de este forma constituirse el "saber" como algo útil, es decir, bueno. Pero esto es posible porque solo un entendido "sabe cómo están dispuestas las cosas mismas" (459c), y no califica el que no es capaz "de dar cuenta de qué es según su naturaleza todo aquello que él aporta a lo que aporta, de tal modo que puede hacer explícito el para qué de todo ello" (465 a1-5). Por tanto, esta forma benéfica de referencia a objetos está caracterizada por una necesaria "veracidad" (alêtheia) y "racionalidad", esto último en el sentido preciso de ser capaz de hacer explícita (echein logon) la "naturaleza" y las "causas" de algo.

El pasaje de Laques es decisivo en otro respecto. Pues es justamente aquí donde aparece con total nitidez cuál es esa condición con la que debe cumplir un entendido en algo. Dice Sócrates en este diálogo: "Si conocemos de algo que presentándose en otra cosa la hace mejor al presentarse en ella y somos capaces de hacer que esto se presente en ella, es evidente que sabemos qué es ello mismo como para hacernos consejeros acerca de cómo alguien podrá adquirirlo de la forma más rápida y mejor" (189 e3-190 a). Sócrates, como ya lo mencionábamos antes, ejemplifica estas afirmaciones con

se quiere, a un uso "fenomenológico", en donde "intencional" apunta a la forma de relación implicada en un determinado comportamiento. Así, por ejemplo, un comportamiento como el "saber" es formalmente diferente al de una "destreza", no por su referencia a distintos "objetos", sino en la medida en que su forma de relacionarse con ellos (que pueden ser extensionalmente idénticos; por ejemplo, la comida en el caso de los médicos y de los cocineros) depende en cada caso de dos respectos ontológicamente diferentes ("realidad"/ "apariencia"). El punto está en que incide en la diferencia de la forma de relación, sin que por ello tenga que suponerse conjuntos diferentes de objetos. Con "extensionalmente" me refiero directamente al conjunto de objetos dados y dables en función de un campo objetual determinado. 
el arte del médico. En efecto, el médico conoce y comprende (epistasthai) cuándo, en qué medida y de qué forma la vista es buena. A su vez, es capaz de establecer las condiciones mediante las cuales se produce efectivamente la vista. Y también es capaz de aconsejar y enseñar a otro cuál es la mejor manera de mantenerla en buenas condiciones o recuperarla. Pero él conoce y es capaz de todo esto porque sabe (eidênai) desde un inicio y en el fondo qué es (ti pote estin) la vista. El pasaje está articulado de tal forma que todas estas facultades cognitivas y productivas de un "saber" tienen su fundamento en el saber qué-es algo. Si justamente consideramos que el arte médico es análogo a un posible saber de las virtudes, esto es así porque la condición primaria de todo saber en general consiste en la posibilidad de delimitar su campo objetual y, en consecuencia, hacer de esta delimitación el fundamento de su referencia a este todo de objetos. Y justamente en cuanto suponemos que quien sabe, sabe ("tiene visto") en el fondo qué es eso, es que podemos exigir que lo exprese y articule en palabras, que lo defina: "de lo que sabemos [qué es], podemos decir qué es" (190c4).

La aporía de un "saber del saber"

No es posible en el presente contexto ahondar en la arquitectura interna de estas determinaciones del saber o, lo que sería más necesario, en las condiciones ontológicas en que Platón plantea las posibilidades adecuadas de responder a la pregunta "¿qué es ...?" "11. Por ahora, más bien, quisiera que reconsideráramos a la luz de lo anterior lo que al comienzo afirmábamos de modo tentativo, a saber, que una posición como la filosófica supone un "saber del saber". Esto concierne a una peculiar paradoja que Platón desarrolla en el Cármides y que toca a la posibilidad de que el "saber" pueda convertirse en objeto para el saber.

El diálogo constituye el intento por determinar lo que sea la sôfrosunê, lo que se traduce habitualmente en nuestra lengua por moderación o temperancia ${ }^{12}$. Sin embargo, el diálogo con Critias llega a un punto en el que cualquier connotación anímica que tenga esta palabra queda abandonada por una dirección que puede parecer en principio un tanto extraña. En efecto, Sócrates reformula la respuesta del sofista (la sôfrosunê es conocimiento de sí mismo) en el siguiente sentido: "Sólo el sensato [sôfrôn] se conocerá a sí mismo y será capaz de discernir realmente lo que sabe y lo que no sabe, y de la misma manera podrá investigar qué es lo que cada uno de los otros sabe y cree

11 Es importante el esfuerzo que a este respecto ha hecho P. Sandoval (2003) influenciado claramente por el pensamiento de Heidegger.

12 Sigo aquí la traducción por la que ha optado Calone et al. (Platón 2000, p. 200). A pesar de que en principio traducir sôfrosunê por "sensatez" parece extraño, el contexto en que rápidamente se ubica la discusión justifica parcialmente esta decisión más "intelectualista", aun cuando con ello se pierdan irremediablemente ciertas resonancias que son más habituales en la palabra griega. 
saber cuando sabe algo, y además qué es lo que cree saber y no lo sabe" $(167 \text { a- } 8)^{13}$. Es innegable el parentesco que hay entre esta formulación y lo que se le atribuye a Sócrates en la "Apología". Ahora bien, no nos ocuparemos aquí del problema de si esto califica o no como definición de la sôfrosunê, sino que lo importante estriba en la postulación de un saber como éste y, aunque esto Platón no lo afirme, en qué medida se da aquí una forma de autointerpretación de la propia filosofía que sea legítima.

Dos aspectos son los que están contenidos en la formulación anterior. Primero, la posibilidad de que haya este peculiarísimo saber "referido a sí mismo"; pero en segundo lugar, que por ello mismo refiera a su vez al resto de todos los otros saberes. Este último aspecto conforma una serie de posibilidades que rebasan la mera formalidad de "un saber del saber". Pues, como lo expresa Sócrates hacia el final de este diálogo (172d2-5), éste sería un "bien inmenso", pues "bajo su vigilancia" podríamos discernir cuáles son efectivamente los saberes y quiénes realmente los desarrollan. De esta forma, el "saber del saber" es un bien inmenso porque nos permitiría "desechar el error" y "vivir rectamente y felices" tanto en nuestras casas como en la ciudades si alguien tal llegase a gobernar en ellas.

Ahora bien, lo que se pone en cuestión es la posibilidad misma de que exista un tal saber y, como consecuencia de esta problematización, el hecho de que constituya un "bien inmenso" (167 a9-b4). Lo interesante de la discusión es que, aun cuando no llega a una negación absoluta de esta posibilidad, sino a un razonable escepticismo (175 a 7-175d3), el argumento pone al descubierto el estatuto ontológico del saber en general, apoyado este argumento en una analogía que abarca tanto el resto de los saberes determinados, pero también otro tipo de facultades (perceptuales y anímicas) (167b4168e). El punto es el siguiente: lo propio de todo saber, de toda facultad perceptiva o de toda disposición anímica es que pensados como referencia a algo (un objeto) son "de" eso (el arte de calcular es de los números, el ver es de lo visible, el temor es de lo temible) y esto implica que difieren de ello en cuanto a su ser (ousia) (168d2). Ser referencia significa estar referido a algo ontológicamente diferente (allos): el cálculo es de los números en cuanto son otros que el cálculo, el ver es de lo visible en cuanto es algo de naturaleza diferente del acto de ver. A este modo de ser de lo referencial lo llama Platón aquí "poder" (dunamis) (168d2)14. Pensado desde su ser, el "saber" no puede constituirse como "autorreferente", y la prueba de esto es que ninguno de los saberes, ni de las facultades perceptuales ni las disposiciones anímicas puede ser en el modo de una autorreferencia. Y aun cuando uno pudiese conceder que se da este único caso en que un poder se refiere a su "objeto" por "reflexión", tampoco este

13 En otros pasajes aparece reformulada esta compleja "definición" de la sôfrosunê: 166 e8$167^{\mathrm{a}}, 167$ b-3; en una peculiar restricción de lo mismo en 170 d-3.

14 Esta misma caracterización ontológica de disposiciones cognitivas en términos de "poder" aparece en un decisivo pasaje Politeia (477 c ss.). Precisamente esta determinación es establecida con respecto a la diferenciación entre episteme y doxa, lo que implica a su vez una diferenciación de sus respectivos dominios ontológicamente modulados (ser-ser y no ser). 
saber tendría la utilidad que se supone. Pues de los otros saberes no sabría más que "que saben o no saben", pero sería inaccesible para él eso que en cada caso se pone en juego como objeto determinado. Y sin esta relación a los objetos mismos no se tiene ninguna competencia para discernir la autenticidad del resto de los saberes y por tanto la función de vigilante es casi nula. No es fundado en este tipo peculiar de saber desde donde se puede decidir si, por ejemplo, un médico es el idóneo para tal función o de si estamos verdaderamente en presencia de un médico (171 a-c7).

Tal vez la paradoja que mencionaba antes no esté totalmente a la vista. Tal vez si la formuláramos en una pregunta ella aparecería con mayor claridad. Pues si efectivamente seguimos el planteamiento en su núcleo central, entonces resulta que Platón está indicando que el saber mismo no se puede constituir el mismo en un nuevo objeto del saber, y menos aún constituirse desde aquí en una "ciencia del saber" con pretensiones de ciencia suma. Entonces puede ser inevitable preguntar: ¿desde dónde surgen todas estas notas esenciales que a todas luces deben ser atribuidas al saber como tal? Ciertamente, tal como lo mencionábamos antes, no puede ser desde un afán meramente "epistemológico", si con esto se entiende justamente el hacer del conocimiento, y en ello el conocimiento científico, un "objeto de conocimiento". Pero si el poner estos caracteres de realce no está motivado por esto, ¿de dónde entonces?

Lo notable de este diálogo es que a pesar de todas las poderosas objeciones que plantea Sócrates, encontramos un cierto pasaje que, así como al paso, salva en parte este "saber del saber y del no saber". Es cierto, Platón nos da una versión bastante más modesta de éste que el de una ciencia suprema y rectora, a la que sin embargo no renunciará en desarrollos posteriores ${ }^{15}$. Esto es lo que hacia el final del diálogo aparece en boca de Sócrates:

¿Acaso....este saber que es experto en el saber y la ignorancia no posee más de bueno que quien lo posee aprende más fácilmente todo lo que, por lo demás está dispuesto a aprender y que todo le aparece más claro, porque al lado de lo aprendido, ve por añadidura el saber mismo? ¿Y no juzgará a los otros más exactamente en eso que él mismo haya aprendido, mientras que querer juzgar a otros, sin haberlo aprendido, será hacerlo más floja y malamente? Así pues querido amigo, ¿no será algo así el provecho que se saca de este saber, y lo que pasa es que nosotros hemos tenido puesta la mirada en algo mayor y buscamos algo mayor de lo que en verdad es?(172b-c3).

Varias conclusiones se pueden sacar de este pasaje, no sin una fuerte dosis de esfuerzo interpretativo. En primer lugar, destaquemos de nuevo la modestia en que parece plausible al menos un saber así. Aquí habla una pretensión "menor"que la que anima en esa otra

15 Con esto me refiero a que, ya en este diálogo (174 e-2), se esboza la posibilidad de una "ciencia rectora", pero en términos de una "epstemê peri to agathon" (ciencia acerca de lo bueno). Ciertamente con ello está en ciernes el "rey-filósofo" de la Politeia. Lo que cabe destacar en ambos casos es que la base desde la que se erige un saber en calidad rector no es meramente "epistemológica", sino debido a su orientación hacia el "bien". 
posibilidad que pretende que por el solo hecho de estar "referido a sí mismo" tiene ya por eso completa jurisdicción sobre los "conocimientos" de aquellos otros saberes que están directamente referidos a sus objetos correspondientes (las ciencias específicas, diríamos nosotros), para erigirse desde aquí como una "ciencia suprema y rectora". Pero, en segundo lugar, no se renuncia con ello a tener una verdadera disposición "científica". No, por cierto, como pasa con el resto de los "saberes"únicamente porque esté dirigido de forma directa a un campo objetual (aunque no esté tampoco excluido de plano la posible referencia a algún o algunos de ellos), sino porque en este saber se da una peculiar acentuación del saber en cuanto es poder y, por eso, el hecho de que es algo tal que puede ser llevado a cabo de forma más o menos plena en cuanto es este poder. Esto es lo que, a mi juicio, habla en la estrecha vinculación entre "saber" y "aprender"; es decir, precisamente en este saber hay un "más" (más fácilmente y más claramente/ raon kai enargestera) en cuanto se acentúa y se ejercita la capacidad y el proceso de aprender. "Saber" es en sentido esencial una práctica que se pone a prueba y se lleva a cabo constantemente. Por ello mismo, en tercer lugar, en esta forma activa de entender el saber se conforma un modo de vivir que nos pone constantemente en guardia contra esa ignorancia pertinaz que pretende saber lo que no sabe, incluso "los más elevados asuntos humanos", en tanto cree tener una posición asegurada acerca de ello. Precisamente frente a esto, este saber está dispuesto a ponerlo todo en cuestión de nuevo para aprenderlo cada vez más claramente.

Para finalizar quisiera responder, por lo menos de manera aproximada, a la última pregunta plateada; a saber, de dónde provienen los caracteres del saber destacados más arriba. Esto ya estaba mencionado antes, pero ahora es el momento de decirlo con mayor claridad. No es motivado por una preocupación epistemológica que surge esta conciencia acerca del propio saber, sino que está movido por lo que nosotros malamente reconocemos bajo los nombres de "moral" o "ética". Malamente, pues ni Platón ni Sócrates conocieron algo como lo que mentamos con estos nombres: una cierta región de objetos a los que refiere una disciplina específica del quehacer científico-filosófico. Más bien los rasgos señalados, si cabe asegurar que se hacen visibles desde un "saber referido a sí mismo", lo hacen porque este saber se conforma como una forma de vida cuya preocupación principal es la de volverse clara con respecto al mejor modo de vivir y en eso mismo llevarse a cabo constantemente. En este sentido, la figura de Sócrates constituye el paradigma del "sabio", porque antes de toda referencia a objetos (aunque esto no signifique excluirlos), lleva a cabo como su propio vivir los caracteres más íntimos del saber.

Referencias bibliográficas

Platón

Platón (1977), Werke in Acht Bänden/Griechisch und Deutsch, Erster Band. Traducción de F. Schleiermacher. Darmstadt: Wissenschaftliche Buchgesellschaft. 
(1923), Ouevres Complète, Tome III, $1^{\circ}$ Partie. Texto establecido y traducido por Alfred Croiset. Paris: Les Belles Lettres.

(2002), Apología de Sócrates. Traducción de Alejandro Vigo. Santiago de Chile: Editorial Universitaria.

(2000), Diálogos, I. Traducción de J. Calone et al. Madrid: Gredos.

(1949), La República,Tomos II y III. Traducción de J. M Pabón et al. Madrid: Instituto de Estudios Políticos.

OTROS AUTORES

Gómez-Lobo, A. (1998), La ética de Sócrates. Santiago de Chile: Andrés Bello.

Guthrie, W. K. Ch. (1998), Historia de la filosofía griega. Vol. IV: Platón, el hombre y sus diálogos, primera época. Madrid: Gredos.

Heidegger, M. (1985), Gesamtausgabe, Band 61. Frankfurt am Main: Vittorio Klostermann.

Kahn, Ch. (1996), Plato and the Socratic dialogues. Cambridge: Cambridge University Press.

Sandoval, P. (2003), La determinación del ser en el pensar temprano de Platón. Tesis para optar al grado de Magíster. Santiago de Chile: Universidad de Chile.

Vlastos, G. (1991), Socrates, ironist and moral philosopher. Cambridge: Cambridge University Press. 